und von chemischen Analyseergebnissen ausgehend zu neuen physiologischen Problemstellungen zu gelangen. Uber Studien am Hämoglobin wurde er dann in dasjenige Arbeitsgebiet geführt, auf dem er seine großen Erfolge errang, nämlich die Eiweißchemie und die mit ihr zusammenhängenden biochemischen und physiologischen Probleme. Die hierzu notwendigen chemischen Grundlagen erwarb er sich bei Emil $F$ is cher, in dessen Instiut er von 1902-1908 arbeitete. Von der Chemie der Aminosäuren ausgehend, an deren Auffindung und Konstitutionsermittlung er maßgebend beteiligt war, befaßte er sich mit der Struktur der Eiweißkörper und stellte dabei als einer der Ersten die allerdings bis heute noch nicht endgültig geklärte Frage der ringförmigen Anordnung von Aminosäuren im छiiweißmolekül zur Erörterung. Fragen des Aufbaues und Abbaues von Eiweißkörpern führten ihn dann zur Verdauungsphysiologie und zum Gebiete der Fermente, speziell dem der proteinasen einschließlich der $\mathrm{Ab}$ wehrproteinasen. Seine Arbeiten auf dem Gebiet der Ernährungsphysiologie, besonders die Ergebnisse seiner Fütterungsversuche an Hunden mit Aminosäuren, haisen ebenso wie seine Untersuchungen über den Gesamtstoffwechsel und die Stoffwechselregulation durch Hormone und Vitamine in weiten Kreisen Beachtung gefunden. Aut dem Gebiet der Wirkstoffe und deren Eingreifen in den Gestamtstoffwechsel oder in Teilphasen des Stoffwechsels hat er in besonders vorbildlicher Weise die chemische und physiologische Betrachtungsart und Methodik vereinigt. Man darf ihn wohl heute als den letzten der deutschen Physiologen bezeichnen, der noch in der Lage ist, die beiden nunmehr getrennten Arbeitsgebiete der Physiologie und physiologischen Chemie in sich zu vereinigen und $z u$ beherrschen.

Indessen sind die Verdienste Abderhaldens durch seine vielseitigen und bedeutsamen Forschungsarbeiten keineswegs erschöpft. Sein Bestreben nach Universalität gepaart mit einer bewunderswerten Aktivität veraniaßte ihn, sich einer umfangreichen literarischen Tätigkeit zu widmen. Bekannt sind vor allem die groß angelegten, von ihm redigierten Sammelwerke, das "Handbuch der biologischen Arbeitsmethoden" sowie das "Biochemische Handlexikon", während seine Lehrbücher der Physiologie und der physiologischen Chemie sowie sein "Physiologisches Praktikum" Zeugnis ablegen von seiner Fähígkeit, auch als akademischer Lehrer sein weites Fachgebiet übersichtlich darzustellen. Bei einer Reihe angesehener Fachzeitschriften beteiligte er sich als Herausgeber oder Mitherausgeber; mit besonderer Liebe und Hingabe widmete er sich aber der "Deutschen Akademie der Naturforscher" in Halle, deren Präsident er lange Jahre hindurch, bis zur unfreiwilligen Rückkehr in seine alte Heimat, war.

H. J. Deuticke.

\section{Adolf Pascher}

A d o If $\mathrm{Pas}$ cher wurde am 31. Mai 1881 in Tusset, einem Dörfchen im südlichsten Böhmerwald, geboren, besuchte hier und in dem benachbarten Mugrau die Volksschule, in Krumau das Gymnasium, studierte dann in Piag Naturwissenschaften, und wirkte daselbst als Forscher, Lehrer und wissenschaftlicher Organisator seit 1933 als Ordinarius und Direktor des Botanischen Institutes und Gartens der Universität - bis zu seinem tragischen Tode im Frühjahr 1945 nach der Einnahme Prags.

Dieser äußerlich stille Lebenslauf war von höchster geistiger-Aktivität und Agilität erfüllt. Auf ungezählten Reisen und Fußwanderungen und bei Aufenthalten an fremden Forschungsstätten erschloB $\mathrm{Pascher}$ in einzigartiger Weise der modemen Forschung die Protistenwelt und gewann von dieser Seite her die Unterlagen zur Behandlung allgemeiner biologischer Probleme. $\mathrm{Zu}$ einem hervorragenden Formensehen und Formengedächtnis gesellte sich eine besondere Art künstlerischer Phantasie, die ihn befähigte, über Floristik und formale Systematik weit hinauszugreifen und zu einer lebendigen Fruchtbarmachung des Gesehenen für die Gesamtwissenschaft zu gelangen. Pascher hat nicht nur eine kaum übersehbare Menge neuer Gattungen und Arten aufgefunden, mustergültig beschrieben, ihre Entwicklungsgeschichte geklärt u. dgl. mehr; er hat vielmehr solche aufgefunden und erforscht, die außer für den Spezialforscher auch von allgemeinem Interesse sind, die Vertreter morphologisch und physiologisch neuer Typen darstellen, neuartige Organisationen aufweisen, wichtige neue phylogenetische Einblicke gewähren, in ökologischer Hinsicht bemerkenswert sind oder für die Symbioseforschung oder sonstwie Bedeutung besitzen. Alle Einzelbeobachtungen wurden bis in die letzten Konsequenzen ausgewertet und in einen großen Zusammenhang gestellt. So konnte $\mathrm{Pasch}$ er der Protistenkunde eine neue Grundlage geben und sie selbst als Glied allgemeinster cytologischer Forschung behandeln. Es ist kein Zufall, daß $\mathrm{Pa} s \mathrm{ch}$ e r auch die ersten erfolgreichen Versuche über haploide Vererbung durchgeführt und damit den ersten Beweis für die Richtigkeit der Mendelschen Grundannahme erbracht hat (1913, veröff. 1916).

Die Ergebnisse seiner wissenschaftlichen Tätigkeit liegen in zahIreichen speziellen und allgemeinen Veröffentlichungen vor. Sie zeichnen sich äußerlich durch die fesselnde und anregende Darstellung und durch die souveräne Behandlung des Jllustrationsmaterials aus. Beispielgebend, erstmalig und unerreicht ist die Sammiung der von $\mathrm{P}$ a s $\mathrm{ch}$ er herausgegebenen Monographien, die unter dem Namen der „P a s cher schen Süßwasserflora" bekannt sind.

DaB eine so reiche Forschernatur auch andere Interessen - wissenschaftliche wie höchst persönliche, so auf künstlerischem Gebiet - besessen hat, ist selbstverständlich Eine von seiner Hand wenige Monate vor seinem Tode geschriebene Selbstbiographie gibt hierüber Aufschluß jenen, die ihn nicht selbst gekannt haben.

Joseph Barcroft T.

L. Geitler.

Am 21. 3. 1947 starb in Cambridge der weltbekannte physiologe Sir $J$ o seph Bar C r of t. Er wurde 1872 in York geboren, er war Fellow des Kings College in Cambridge, Fellow der Royal Society und erhielt 1943 die Copley Medaille, die höchste Auszeichnung, welche die Royal Society zu vergeben hat.

Sein Hauptwerk bis zur Zeit nach dem ersten Weltkrieg war die Klärung der Sauerstofftransportfunktion des Blutes, dessen monographische Darstellung auch in Deutschland unter dem Titel: "Die Atmungsfuriktion des Blutes" erschien. Im Verlaufe seiner weiteren Arbeiten uber das Problem der Anpassung und Regulationen entdeckte er die Notwendigkeit und Tatsache der "Blutspeicher". In den letzten Jahren waren es seine Unterspercher über das embryonale Hämoglobinsystem und über das nervöse Atemzentrum, die unser Wissen ungeahnt erweitert haben.

Kein Physiologe, ja kaum ein Arzt blieb vom Wirken und den wissenschaftlichen Ergebnissen dieses Mannes unbeeinflußt, der nicht nur im Laboratorium, im Tierexperiment und Selbstversuch, sondern auch auf zahlreichen Bergexpeditionen mit genialem Blick und oft verblüffend einfacher Methodik das Lebensgeschehen zu ergründen suchte.

L. V. K.

\section{Berichtigung.}

In dem Nachruf auf $F$. $v$. We $t$ t s tein ist zu verbessern: S. 97, linke Spalte, 18. Zeile von unten: "entdeckt im 20. Jahrhundert" statt "entdeckt, im 19. Jahrhundert". S. 97, rechte Spalte, gegen unten "auch die zu Klebs an" statt, ,auf"; ebenda 3. Zeile von unten "vor niemandem" statt "niemanden". S. 100 rechts 1. Zeile "Wasen" (so bei Kerner) "des Blaser" statt "Wiesen des Blasers". In den Versen aus Goethes Achilleis Allen Künftigen auf" statt ,auch". Endlich am SchluB

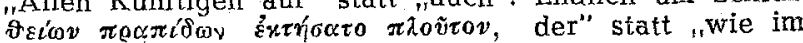
Motto am Anfang".
O. Renner 